\title{
Application of the boundary elements method to 3-D radiation problems
}

\author{
J. Abad, N. Duffo, J.M. Rius, J.C. Cruellas \\ Dpto Teoría de la Señal y Comunicaciones, Grupo A.M.R. \\ E.T.S.I. de Telecomunicaciones de Barcelona. U.P.C \\ Apdo. 30002, 08080 Barcelona Telf 93/401.72.61
}

\section{Abstract}

In order to analyze the electromagnetic fields generated by perfectly conducting bodies composed by interconnected wires and solid objects, whichever its geometry, the Boundary Element Method is applied. The basic formulation and the associated items are commented. Results of problems of radiation are given and compared with available analytic solutions.

\section{Introduction}

There are many three dimensional electromagnetic problems that can be solved by using the Boundary Elements Method. In this paper we consider the study of radiation and scattering properties of interconnected closed surfaces and thin wire antennas. Due to the fact that the formulation employed in the study of thin wires and closed surfaces are usually fairly different, it becomes necessary to build a starting strategy which combine both cases. The problem can be partitioned in the four following items.

1. Mutual interaction among nodes in the wire.

2. Mutual interaction among nodes on the surface.

3. Cross interaction among wire's nodes and surface's nodes.

4. Cross interaction among surface's nodes and wire's nodes.

The first approach to the problem consists of solving independently each item by proposing a suitable integral equation and solving it by using the Boundary Element Method. Afterwards, it will be possible to deal with the general case.

\section{Formulation and Integral Equations}

\section{Radiation of perfectly conducting bodies}

Radiation of thin wires and closed surfaces can be handled in the same way by using the well known radiation integrals showed in Ballanis [1] expressed in their cartesian coordinates form

$$
\begin{aligned}
& H_{A x}=\frac{1}{4 \pi} \iiint_{V}\left[\left(z-z^{\prime}\right) J_{y}-\left(y-y^{\prime}\right) J_{z}\right] \frac{1+j \beta R}{R^{3}} e^{-j \beta R} d x^{\prime} d y^{\prime} d z^{\prime} \\
& H_{A y}=\frac{1}{4 \pi} \iiint_{V}\left[(x-x) J_{z}-\left(z-z^{\prime}\right) J_{x}\right] \frac{1+j \beta R}{R^{3}} e^{-j \beta R} d x^{\prime} d y^{\prime} d z^{\prime} \\
& H_{A z}=\frac{1}{4 \pi} \iiint_{V}\left[(y-y) J_{x}-\left(x-x^{\prime}\right) J_{y}\right] \frac{1+j \beta R}{R^{3}} e^{-j \beta R} d x^{\prime} d y^{\prime} d z^{\prime}
\end{aligned}
$$

$$
\begin{aligned}
& E_{A x}=-\frac{j \eta}{4 \pi \beta} \iiint_{V}\left\{G_{1} J_{x}+(x-x) G_{2}\left[(x-x) J_{x}+(y-y) J_{y}+\left(z-z^{\prime}\right) J_{z}\right]\right\} e^{-j \beta R} d x^{\prime} d y^{\prime} d z^{\prime} \\
& E_{A y}=-\frac{j \eta}{4 \pi \beta} \iiint_{V}\left\{G_{1} J_{y}+(y-y) G_{2}\left\{(x-x) J_{x}+(y-y) J_{y}+\left(z-z^{\prime}\right) J_{z}\right] e^{-j \beta R} d x^{\prime} d y^{\prime} d z^{\prime}\right. \\
& E_{A z}=-\frac{j \eta}{4 \pi \beta} \iiint_{V}\left\{G_{1} J_{z}+\left(z-z^{\prime}\right) G_{2}\left[\left(x-x^{\prime}\right) J_{x}+(y-y) J_{y}+\left(z-z^{\prime}\right) J_{z}\right]\right\} e^{-j \beta R} d x^{\prime} d y^{\prime} d z^{\prime}
\end{aligned}
$$

which allows to evaluate the electric and magnetic fields generated by a current distribution on the wire or on closed surfaces.
On the other hand, an alternative expression developed from the Stratton Chu integral equation can be found in terms of fields

$$
\begin{aligned}
& E=-\frac{T}{4 \pi} \int_{s_{1}+s}\left\{j \omega \mu\left(\hat{n}^{\prime} \times A\right) \varphi-\left(\hat{n}^{\prime} \times E\right) \times \nabla^{\prime} \varphi-\left(\hat{n}^{\prime} \cdot E\right) \nabla^{\prime} \varphi\right\} d s^{\prime} \\
& A=-\frac{T}{4 \pi} \int_{s_{1}+s}\left\{j \omega \varepsilon\left(\hat{n}^{\prime} \times E\right) \varphi-\left(\hat{n}^{\prime} \times A\right) \times \nabla^{\prime} \varphi-\left(\hat{n}^{\prime} \cdot A\right) \nabla^{\prime} \varphi\right\} d s^{\prime}
\end{aligned}
$$

where $\frac{1}{T}=\left(1-\frac{\Omega}{4 \pi}\right)$ is the normalized internal solid angle of the surface.

Thin wire's equation

In order to calculate the current distribution on the wire, equation (2) is used.

By imposing the boundary condition $-\hat{l} \cdot \vec{E}^{i}=\hat{l} \cdot \vec{E}^{s}$ on the cylindrical surface of the wire and particularizing for wires in the $\mathrm{z}$ axis, the following expression is obtained:

Where

$$
E_{z}^{i}=-\frac{j \eta}{4 \pi \beta} \int_{1}\left[G_{1}+(z-z)^{2} G_{2}\right] I e^{-j \beta R} d l^{\prime}
$$

$$
G_{1}=\frac{-1-j \beta R+\beta^{2} R^{2}}{R^{3}} ; \quad G_{2}=\frac{3+3 j \beta R-\beta^{2} R^{2}}{R^{5}}
$$

The current is expressed, as in the Finite Element Method, in terms of the following expansion:

$$
I=\sum_{p} I_{p} \phi_{p}(\eta)
$$

With the variable discretized, a system of $\mathrm{N}$ equations is enforced, whose unknowns are the nodal values of the current distribution and whose matrix expression is:

$$
[-E]_{z}=[W W][I]
$$

In the matrix notation used henceforward, the first letter means the source and the second one the destination (W:wire, S:surface).

\section{Surface's equation}

When the object is not a wire but an arbitrary conducting solid, the scattered magnetic field must be calculated by using equation (3). This problem was studied by Paulsen et al.[2] The boundary condition $\hat{n} x E^{i}=-\hat{n} x E^{s}$ is forced on the surface, which is discretized by using Finite Element Method second order isoparametric elements. The fields are approximated on each element by using the same Lagrangian shape functions used in the surface discretization:

$$
F(\xi, \eta)=\sum_{l} F_{l} \Psi_{l}(\xi, \eta)
$$

In this way, a NsxNs equation system is obtained, whose unknowns are the nodal values of the scattered magnetic field:

$$
[S S][H \tau]=-[C]\left[\hat{n} x E^{\dagger}\right]
$$

It is important to emphasize that the equation is written in terms of scattered and incident fields and not current distribution.

\section{Cross interaction among wires and surfaces}

In a complex object, wires and closed surfaces interact: the fields scattered by surfaces are part of the incident fields on wires and the currents on wires generate scattered fields that, in turn, are part of the incident fields on surfaces. This interactions 
among both components of the object are modelled by two equation systems, derived from equations (1) and (2), once the surface and the wire have been discretized and the corresponding unknowns (current distribution) have been interpolated:

$$
\begin{aligned}
& {[S W][J]=\left[E^{J}\right]} \\
& {[W S H]\left[\eta=\left[H^{J}\right]\right.} \\
& {[W S E]\left[\eta=\left[E^{S}\right]\right.}
\end{aligned}
$$

Where $\mathbf{J}$ is the current distribution on the surface. It must be pointed out that in the previous paragraph, the problem on the surface has been formulated in terms of fields instead of currents, which will imply additional work in the assembly phase.

\section{Assembly of the equations system}

The last step consists of building the matrix equation in order to solve the general problem. For the wire the following expression is derived by using equations (8) and (11):

$$
\left[W W[\Pi]=[S W][J]+\left[E_{\text {source }}^{i}\right]\right.
$$

where $E_{\text {source }}^{\mathrm{i}}$ states for the excitation of the system.

Proceeding in a similar way for the surface, the corresponding expression can be found by manipulating equation (10):

$$
[C][n]\left[E_{\text {source }}^{i}+E_{\text {wire }}^{i}\right]=[D]\left[H^{\top}\right]
$$

where $\mathrm{E}_{\mathrm{wire}}^{\mathrm{i}}$ represents the incident field caused by the radiation of the wire and $[n]$ is a matrix whose product with the electric field results in the tangential component to the surface of it.

Finally, substituting (12) into (14) and assembling (13) and (14) yields

$$
\left[\begin{array}{cc}
{[W W} & -[S W][n] \\
{[C][n][W S E]+[D][W S H]} & -[D]
\end{array}\right]\left[\begin{array}{l}
{[\eta]} \\
{[H]}
\end{array}\right]=\left[\begin{array}{c}
{\left[l \cdot E_{\text {source }}^{i}\right]} \\
-[C][n]\left[E_{\text {source }}^{i}\right]-[D]\left[H_{\text {source }}^{i}\right]
\end{array}\right]
$$

It will be necessary to use an accurate modeling of the source in order to obtain good results. When the currents on the wire and on the surface are known, the radiation pattern and the input impedance can be found.

\section{Results}

The first example we consider is the radiation pattern of a 0.25 $\lambda$ monopole antenna driven against a perfectly conducting sphere of radius $0.25 \lambda .153$ nodes on the surface and 13 nodes on the wire have been considered. In Fig. 1, we compare our boundary element results with results provided by Bolle and Morganstern [3] and Teshe and Neureuther [4]. There is a good agreement among the three results. The solution for that grid took $1200 \mathrm{CPU}$ seconds to compute on a CONVEX C -340 .

The second example problem consists of a right circular cylinder with a monopole antenna. The dimensions of the cylinder and the wire are shown in Fig.2 as well as our boundary element results using 151 nodes on the surface and 13 on the wire, compared with Albertsen [5] results.

\section{Conclusions}

Software which allows to deal with conducting arbitrary objects composed by attached wires and surface, has been developed. Integral equations are derived and treated using Boundary Element Method for isolated parts and interactions among them.

Afterwards these are assembled in an unique equations system whose resolution allows to know current distribution on the wires and surfaces.

Several objects have been analyzed and the results compared with previous references, showing a good agreement with them.

Problems related with source modelling and current continuity have been treated carefully.

\section{Acknowledgement}

This work has been sponsored by the Comision Interministerial de Ciencia y Tecnología (CICYT') under Grant TIC 91/1034

\section{References}

[1] C.A. Balanis, "Advanced Engineering Electromagnetics", Wiley \& Sons. 1989

[2] K.D. Paulsen, D.R. Lynch \& J.W. Strohbehn, "Three Dimensional Finite, Boundary, and Hybrid Element Solutions of the Maxwell Equations for Lossy Dielectric Media", IEEE Trans. Microwave Theory Tech., vol 36, pp. 682-693, April 1988.

[3] D.M. Bolle, M.D. Morganstern. "Monopole and Conic Antennas on Spherical Vehicles", IEEE Trans. on Antennas and Propagation, vol AP-17, pp. 477-484, July 1969.

[4] F.M. Tesche, A.R. Neureuther, "Radiation Patterns for two Monopoles on a Perfectly Conducting Sphere", IEEE Trans. on Antennas and Propagation, September 1970.

[5] N.C. Albertsen, J.E. Hansen, N.E. Jensen, "Computation of Radiation from Wire Antennas on Conducting Bodies", IEEE Trans. on Antennas and Propagation, March 1974.

\section{BOUNDARY ELEMENT METHOD}
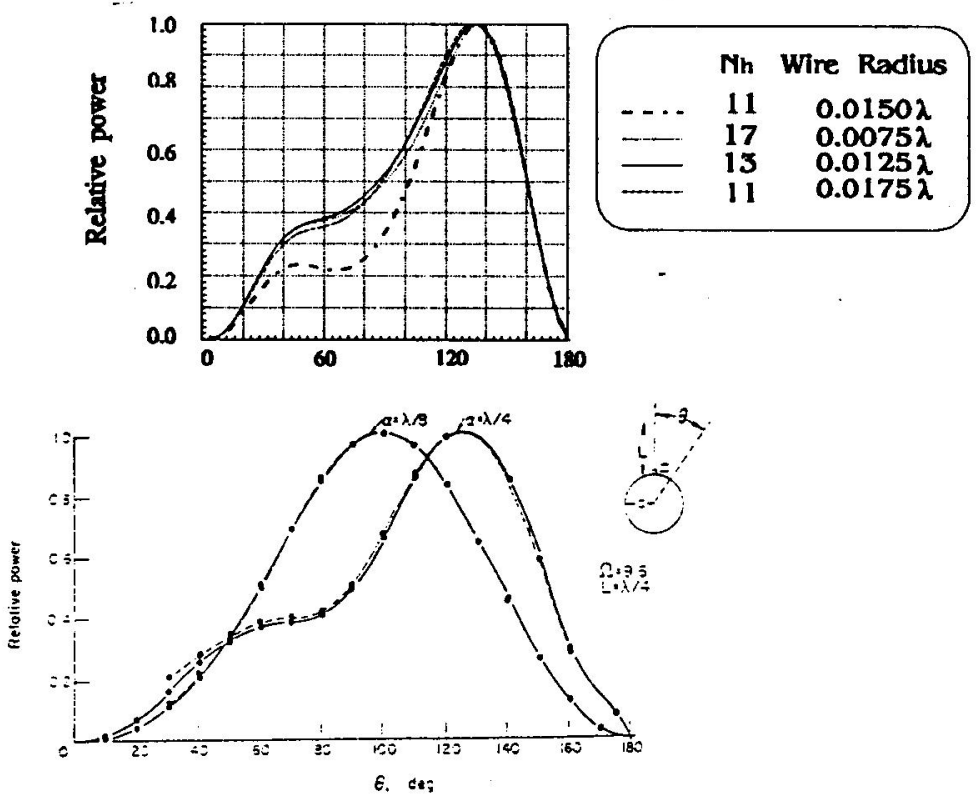

Sphere with monopole

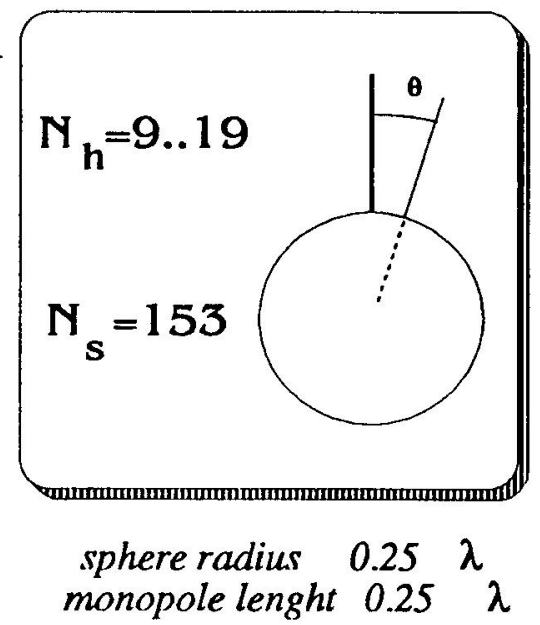

Fig. 1 Sphere with monopole 


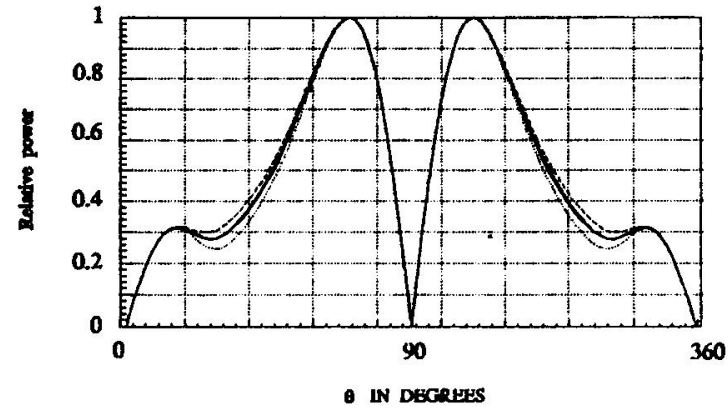

Cilinder with monopole
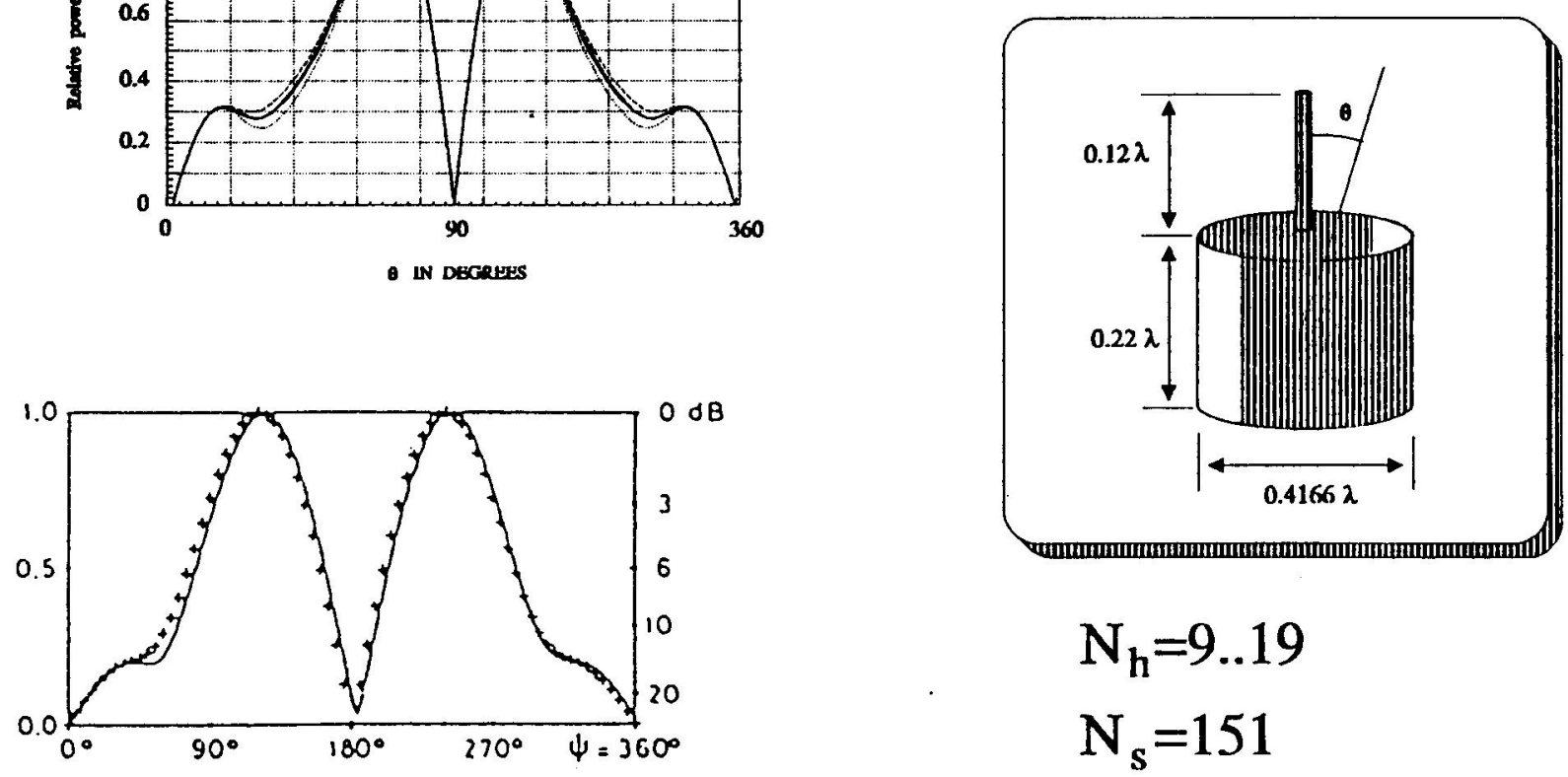

$\mathrm{N}_{\mathrm{h}}=9 . .19$

$\mathrm{N}_{\mathrm{s}}=151$

Fig. 2 Cilinder with monopole 